\title{
Effect of click rate and delay on breakdown of the precedence effect
}

\author{
RACHEL K. CLIFTON and RICHARD L. FREYMAN \\ University of Massachusetts, Amherst, Massachusetts
}

\begin{abstract}
The precedence effect was tested as a function of echo-click delay and click rate after an abrupt switch in location between leading and lagging clicks. Click trains at three rates, $1 / \mathrm{sec}, 2 / \mathrm{sec}$, and 4/sec, with delays ranging between 2 and $20 \mathrm{msec}$, were presented to subjects in an anechoic chamber. Duration of the click train after the switch in location was $12 \mathrm{sec}$, and echo click perceptibility was assessed throughout this period. The number of echo clicks heard was an increasing monotonic function of delay. The subjects reported a "fade-out" of echo clicks after a set number of clicks at each delay, regardless of rate. This result was interpreted as a buildup in inhibition of echoes produced by the ongoing click train. Suppression of echoes was stronger when the leading click originated from the right side than from the left side.
\end{abstract}

Under normal room conditions, we localize a sound at its source, while failing to localize or even recognize the presence of numerous reflected sounds from surrounding surfaces. This perceptual phenomenon is known as the precedence effect or "law of the first wavefront" because we weight the original, or first-arriving, sound more heavily than any later-arriving sounds. In a classic study, Wallach, Newman, and Rosenzweig (1949) simulated natural room reverberations by playing the same sound through two loudspeakers, with one output leading the other by a few milliseconds. As the delay between the original sound and the lagging sound was increased, the listener eventually perceived the lagging sound at its locus. Blauert (1983, pp. 224-225) defined the echo threshold as the shortest delay at which a single auditory event breaks apart and the lagging sound is localized in a different direction from the original sound. Echo suppression at short delays is produced by inhibitory processes, probably in the central auditory system (Zurek, 1987).

Recently, Clifton (1987) reported that a breakdown of this echo suppression can be produced if the location of leading and lagging sounds is suddenly switched. The experimental situation was a train of clicks from two loudspeakers placed at $\pm 90^{\circ}$ off the listener's midline, with one output leading the other by about $5 \mathrm{msec}$ (see Figure 1A for diagram). The listener initially localized the clicks solely at the leading side. When the leading and lagging sides were switched within an interclick interval, the listener heard clicks from both loudspeakers for several seconds before echo suppression was reestablished.

This research was supported in part by National Science Foundation Grant BNS-8812543 to R.K.C. and R.L.F., Biomedical Research Support Grant RR07048-21 to R.L.F., and a Research Scientist Award from the National Institute of Mental Health (MH00332) to R.K.C. Please address reprint requests to Rachel Clifton, Department of Psychology, University of Massachusetts, Amherst, MA 01003.
Click rate determined how quickly the precedence effect was reestablished; the faster the rate, the faster echo suppression reappeared. The "fade-out" of the lagging click appears to be due to a buildup of echo suppression that is surprisingly gradual, taking place over several seconds. The buildup of echo suppression is not limited to cases in which suppression is reestablished following a switch in location. Thurlow and Parks (1961) presented click trains to determine echo thresholds, asking subjects to indicate on each trial whether they heard one or two sounds. They reported that many subjects noted that echo suppression did not appear immediately, but took 1 or $2 \mathrm{sec}$ to "build up" when the click rate was $5 / \mathrm{sec}$. These authors speculated that this changing perception was due to "a central build-up of an inhibitory effect" (p. 11). This is the only study, to our knowledge, that used click trains, rather than brief stimuli, in establishing subjects' echo thresholds, a necessary condition for experiencing the buildup of echo suppression. The description of the buildup was reported in Thurlow and Parks' discussion as a casual response during postsession questioning of subjects, and was thus an incidental observation rather than part of the analyzed data.

Clifton's (1987) observations of a "breakdown" in echo suppression following a location switch have at least two aspects that may prove significant for understanding the precedence effect: (1) they suggest that the precedence effect should be conceptualized as a dynamic process that is influenced by previous inhibition and ongoing stimulation, and (2) they describe a procedure that may allow us to track the temporal course of inhibition that produces echo suppression. Although the precedence effect has a long history of experimentation (see Gardner, 1968, for historical review), the mechanisms responsible for it are not well understood. The strength of the precedence effect varies with delay between onsets, intensity of the lagging sound relative to the primary sound (Wallach et al., 1949), the presence of transients (Rakerd \& Hartmann, 


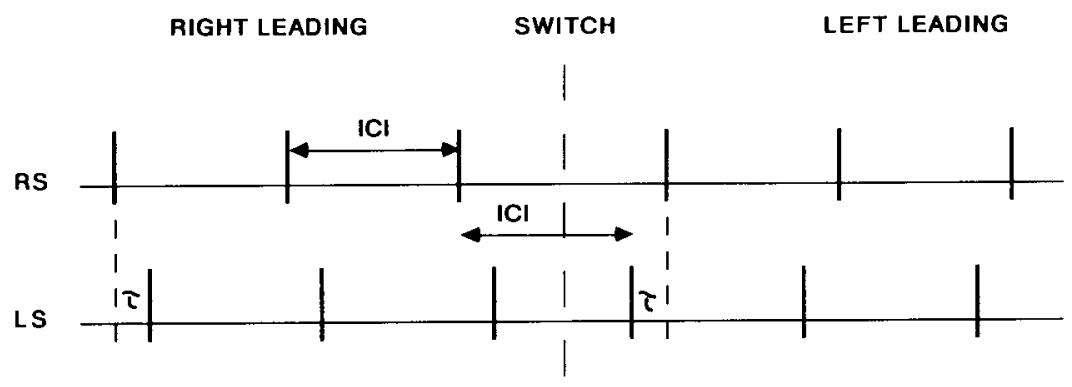

(A)

DESIGN

Click rate
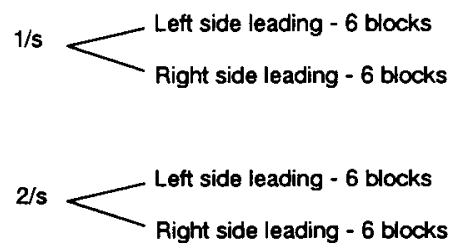

Each block contained

10 trials, one at each

delay plus two control

trials.

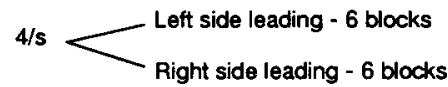

(B)

Figure 1. (A) Schematic diagram of click trains showing the switch in lead-lag loudspeaker locations. Clicks are represented by vertical lines, with RS and LS as right and left loudspeaker outputs, respectively. The delay between outputs (-) varied between 1 and 20 msec, and is not drawn to scale. ICI is the interval between leading clicks and remained constant through the switch in locations. (B) Diagram indicating the trials each subject received at each click rate.

1985), spectral density (Hartmann, 1983), and the nature of the stimulus (e.g., clicks, noise, speech, or music; see Blauert, 1983, for review). Within these variations in echo threshold, the inhibitory process is viewed as relatively static in that no carryover from one auditory event to the next has been postulated. To reveal dynamic processes, a long-duration stimulus that continually evokes inhibition is needed. A train of clicks may be the ideal stimulus, as each auditory event (click) evokes maximal inhibition. Rakerd and Hartmann (1986) found that the precedence effect was maximally invoked when signal onset was instantaneous; echoes were most suppressed when onsets were abrupt.

Clifton (1987) described the existence of the breakdown phenomenon but reported no systematic data. We now present data on echo perceptibility following a switch in lead and lag locations as a function of click rate and delay between onsets. We predicted that the echo click would be heard for longer periods following the switch with longer delays. If the breakdown phenomenon has a continuous rather than an "all-or-none" character, short delays well below echo threshold should result in a shorter period of breakdown in echo suppression. That is, the precedence effect should not be disrupted for very long when delays are short. As delays approach echo threshold, the breakdown period should correspondingly increase. We manipulated click rate at the same rates reported by Clifton (1987) to determine if rate and delay interact.

\section{METHOD}

All sessions were conducted in free field in a $3.1 \times 3.4 \times 3.4 \mathrm{~m}$ anechoic chamber. The subjects sat in a chair, equidistant at $11 / 2 \mathrm{~m}$ from the loudspeakers positioned $90^{\circ}$ to the right and left of midline. The stimuli were trains of click pairs generated on a Zenith Z-100 computer. Pairs of positive electrical pulses $160 \mu \mathrm{sec}$ in duration were created through two-channel digital-to-analog (D/A) conversion of the computer-generated waveform at a $25-\mathrm{kHz}$ rate. The trains of clicks were recorded on two channels of a Teac reel- 
to-reel tape recorder; on playback, the recorded stimuli were amplified by a Marantz amplifier and presented over two Marantz loudspeakers (HD770), which had been carefully matched for frequency response by monitoring their outputs to frequency-swept sinusoids.

The tape recording of clicks from the computer was conducted with one D/A channel leading the other to produce delays between loudspeaker outputs. Delays between leading and lagging clicks, created by using the computer's programmable clock, were 2$9 \mathrm{msec}$, in $1-\mathrm{msec}$ steps, and $15 \mathrm{msec}$. Because all delays were multiples of the $40-\mu \mathrm{sec}$ clock period, error in the delays was much less than $\mathbf{4 0} \mu \mathrm{sec}$. The accuracy of the delays was confirmed through examination of two-channel oscilloscope traces of the D/A outputs. Measurements of the acoustic signal in the anechoic chamber were conducted using a Brüel and Kjaer sound-level meter set on the "impulse" meter response with the microphone at the site of the subject's head. Throughout the experiment, lead and lag clicks were always presented at equivalent levels. The output of each loudspeaker individually was calibrated to be $56 \mathrm{dBC}$, and of both loudspeakers together, $59 \mathrm{dBC}$. The AC output of the SLM was fed to a Tektronix oscilloscope for visual examination of the acoustic click waveforms. The high-amplitude portion of each acoustic click consisted of an initial positive pressure pulse of approximately $1-$ msec duration, followed immediately by a 2 -msec negative spike with a peak amplitude of approximately $70 \%$ of the initial positive pulse. These were followed by a second pair of positive and negative peaks having amplitudes of $15 \%$ and $10 \%$, respectively, of the initial pulse. The total duration of the second set of peaks was approximately 12 msec.

A trial consisted of a click train that began with the leading click from the right loudspeaker and the lagging click from the left (or vice versa). After 5 to $8 \mathrm{sec}$ (randomly varying from trial to trial), the locations of the leading and lagging clicks were switched within a silent interval (see Figure 1A). The click train continued for $12 \mathrm{sec}$ after the switch. Intertrial intervals were $6 \mathrm{sec}$. The timing of click delays, the switch in locations, and intertrial intervals were programmed on the stimulus tape. Throughout the trial, the subject pressed a left button if a click was perceived from the left loudspeaker and a right button if a click was perceived from the right loudspeaker. Both buttons were pressed if clicks were perceived from both locations. Button presses and clicks from the tape recorder were recorded on an Esterline Angus strip chart, running at a speed of $1 \mathrm{~mm} / 1.7 \mathrm{sec}$. The button presses were scored to the nearest $0.5 \mathrm{~mm}$ during the $12 \mathrm{sec}$ following the location switch in leading and lagging clicks. For each trial, a subject's score was the total millimeters a button was pressed on the side of the echo click. The subjects always pressed the button on the leading side, so these data were of interest only insofar as they were evidence of attention to the task.

Five subjects were run, 2 experienced (the authors) and 3 naive. Audiograms showed that all subjects had no more than $10 \mathrm{~dB}$ asymmetry at any frequency from 500 to $8000 \mathrm{~Hz}$, and pure-tone thresholds were $15 \mathrm{~dB}$ HL or better at all frequencies tested. All subjects received the same stimulus conditions but in different randomized orders. Each subject received a total of 360 trials, organized into 36 blocks of trials, 12 blocks at each rate (see Figure 1B for design). Within each rate, there were three random orders of click delays, each order presented four times to compose the 12 blocks at each rate. A block contained 10 randomized trials, one trial at each click delay (2-9 msec) plus two control trials: a single source trial in which only one loudspeaker was activated, and a 15-msec click delay that was expected to be above the subjects' thresholds. The purpose of the control trials was to anchor the subject's perception with clear examples of "no-echo present" and "echopresent" trials. In addition, performance on these trials could be used to evaluate the subject's attention to appropriate aspects of the experiment. Each block contained only one click rate and always began from the same side. Half of the blocks at each rate began with the left loudspeaker leading and right lagging, and half were reversed. The subjects were run for four sessions, one session per day, with nine blocks of trials presented each session.

\section{RESULTS}

Analysis of the data indicated a strong laterality, such that echo suppression was much stronger in several subjects when the leading click originated from the right side than from the left side. This laterality effect was unexpected and will be discussed below. Because the difference between right-leading and left-leading echo thresholds was so pronounced, adding over the two sides would distort the data. Therefore, the major results of the study will be presented for left-leading data only, followed by results comparing the two sides.

The temporal course of inhibition of echo clicks following a switch in lead-lag location is shown in Figure 2. Data are presented for Subjects 1 and 2 at the 1/sec rate for three delays when the leading click originated from the left loudspeaker following the switch. Each numeral on the abscissa corresponds to a 1-sec interval; for example, "1" refers to the interval between 0 and $1 \mathrm{sec}$ following a switch and " 12 " represents the interval between 11 and $12 \mathrm{sec}$. The ordinate shows the percentage of trials on which the lagging click was heard (i.e., the
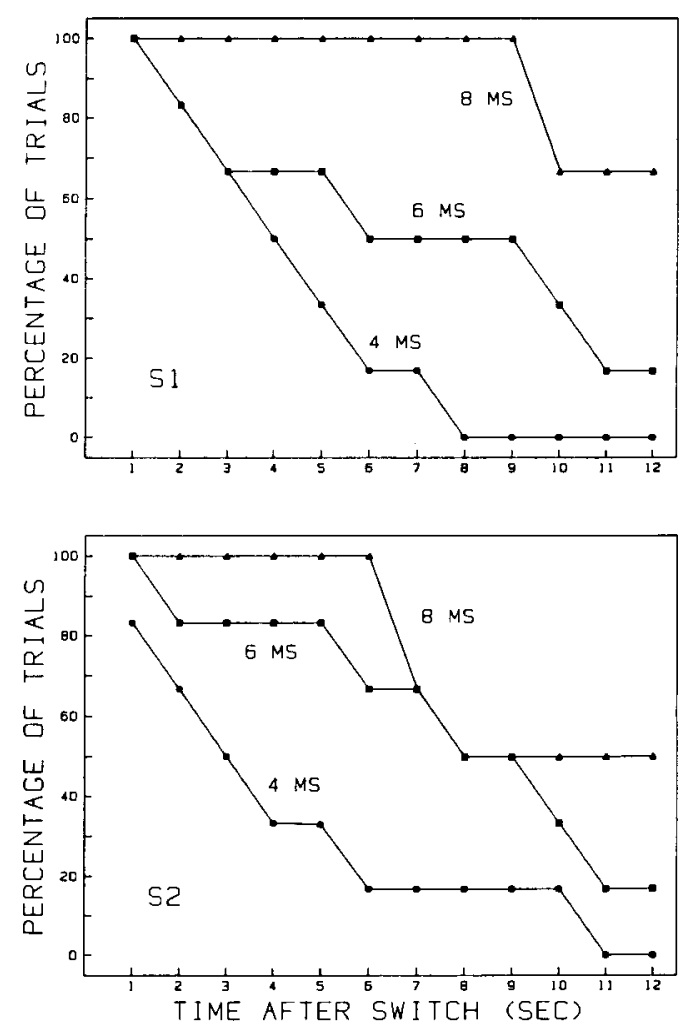

Figure 2. Percentage of trials on which the echo click was heard following a switch in location of leading and lagging clicks. Data are plotted for delays of 4,6 , and $8 \mathrm{msec}$ as a function of time after the switch. Data shown are for a click rate of $1 / \mathrm{sec}$, left side leading, for Subjects 1 and 2 (S1 and S2) in separate panels. 
button was depressed) during each of these 1-sec intervals. For all three lagging-click delays shown, echo clicks were most likely to be heard immediately following the switch, and least likely to be heard at 11-12 sec after the switch. This tendency for subjects to report hearing lagging clicks early, but not late, in the click train following the switch demonstrates the fade-out of the perception of the lagging click.

The frequency with which the lagging click was perceived during any particular time interval after the switch depended strongly on the delay of the lagging click. The effect of delay is shown more comprehensively in Figure 3, which displays left-leading data averaged across the 5 subjects for all delays. The mean number of seconds that lagging clicks were heard following the switch is plotted as a function of delay for the three different click rates. The mean length of time that lagging clicks were heard depended on delay, such that at shorter delays the lagging click either was not heard or faded out quickly, whereas at longer delays the lagging click was heard for a greater portion of the $12 \mathrm{sec}$ of postswitch stimulation. This effect of delay on echo perceptibility during the seconds following the switch would be predicted from the wellestablished effect of delay on echo perceptibility in general. Inhibition is so strong at short delays that the suppression of the lagging click is unlikely to be broken down by a sudden switch in lead and lag locations.

As expected, the subjects did not report hearing the lagging clicks following the switch on single-source control trials, indicating that the perception of clicks from the lagging side after the switch, when reported, was not somehow "imagined"' as a continuation of stimulation from that side which immediately preceded the switch. The responses to the long-delay $(15-\mathrm{msec})$ control trials in Figure 3 were surprising, in that the literature led us to expect that the lagging click would be heard throughout the postswitch period. The two slower rates fulfilled this

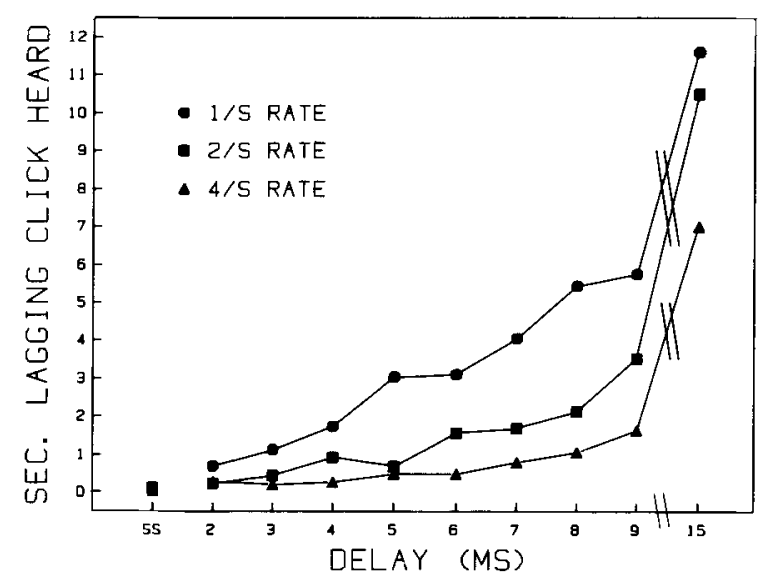

Figure 3. Number of seconds that subjects heard the echo click following a switch in location of leading and lagging clicks. Note that the maximum number of seconds is 12 on each trial, and each datum point is the mean of $\mathbf{3 0}$ trials, 6 for each subject.

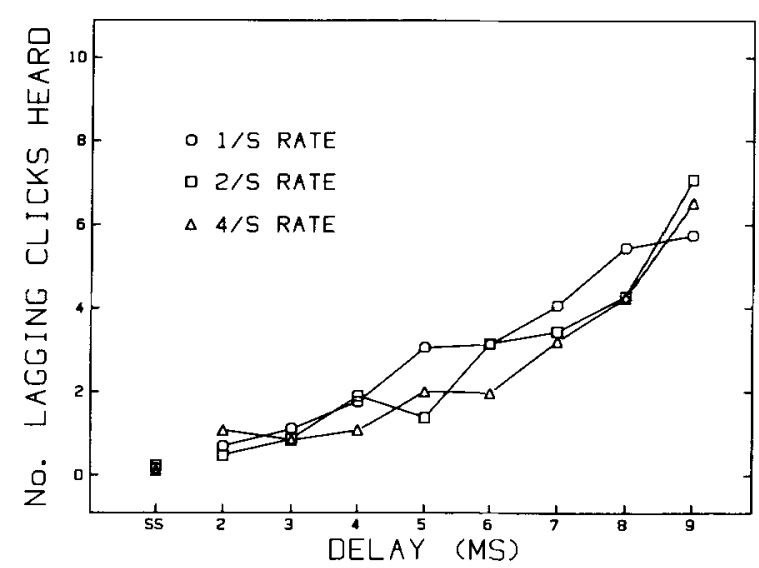

Figure 4. Number of echo clicks that subjects heard following a switch in location of leading and lagging clicks. These are the same data as in Figure 3, replotted with clicks on the ordinate. Note that the longest delay (15 msec) was not plotted because data for the slower rates would be truncated (see text for details).

expectation, but the $4 / \mathrm{sec}$ rate produced inhibition by $7 \mathrm{sec}$.

The effect of click rate on the perception of the echo click was considerable. The data in Figure 3 clearly indicate that the slower the click rate, the longer the lagging click was heard following the switch. This was true for every delay. For example, at a delay of $8 \mathrm{msec}$, clicks were heard for an average of approximately $1 \mathrm{sec}$ for the $4 / \mathrm{sec}$ rate, for about $2 \mathrm{sec}$ for the $2 / \mathrm{sec}$ rate, and for about $5.5 \mathrm{sec}$ for the $1 / \mathrm{sec}$ rate. Although the reasons behind the rate effect are not completely clear, the direction of the effect leads to the suspicion that the fade-out of the echo click's perceptibility may be more related to the number of clicks following the switch than to the length of time after the switch occurred. To evaluate this possibility, the data in Figure 3 were replotted in Figure 4, with the ordinate showing number of clicks following the switch.

As shown in Figure 4, the number of clicks heard before the fade-out was relatively constant and independent of the click rate for a given lagging-click delay. For example, at the 6-msec delay, only two to three clicks were heard after the switch, regardless of the click rate. At the 9-msec delay, six to seven clicks were heard for all three click rates. The 15-msec data (not shown in the figure) are 11.6 clicks at the $1 / \mathrm{sec}$ rate, 21 clicks at the $2 / \mathrm{sec}$ rate, and 28 clicks at the $4 / \mathrm{sec}$ rate. The $12-\mathrm{sec}$ duration of the postswitch click train allowed only 12 clicks to be presented for the $1 / \mathrm{sec}$ rate, whereas 24 were presented for the $2 / \mathrm{sec}$ rate and 48 for the $4 / \mathrm{sec}$ rate. Thus, the $1 / \mathrm{sec}$ mean data at the 15-msec delay are artificially truncated at approximately 12 clicks and cannot be compared fairly with the other rates. The data for the shorter delays shown in Figure 4 suggest that the fade-out of the lagging click is more a function of the number of clicks presented after the switch than of the time elapsed since the switch. The effect of click rate seen in Figure 3 appears to have 
little to do with interclick interval per se; rather, rate seems to be important only to the extent that it determines the amount of time necessary for a critical number of clicks to be presented.

As noted earlier, a strong laterality effect was observed. Unexpectedly, there was greater suppression of echo clicks when the leading click originated from the right side than from the left side. This effect was most dramatic for 3 of the subjects, but was observed to at least some extent in data from all 5 subjects. To exclude the possibility that this side bias was due to artifacts produced by the loudspeakers or to some physical aberration in the room, 2 subjects who showed strong laterality were tested when turned around $180^{\circ}$, reversing the situation from their point of view. This reversal produced no diminution of the effect, confirming its psychological origin. Figure 5 shows 2 subjects with strong laterality plotted for the same delays as in Figure 3 for the $1 / \mathrm{sec}$ rate. In the previous figures, we chose to present data following the switch in location with the left loudspeaker leading because lagging clicks were heard across the range of delays presented. The right-leading data, if plotted on the same graphs as Figures 3 or 4, would show essentially flat functions for all three rates with almost no lagging clicks reported from 2 to $9 \mathrm{msec}$. The only right-leading stimuli-producing responses of more than one or two lagging clicks were those with an echo delay of $15 \mathrm{msec}$. An examination of the three right-leading datum points (one for each rate) at the 15-msec delay revealed the same rate effect obtained in the left-leading data described above; lagging clicks were heard for an average 9.5, 4.8, and $2.3 \mathrm{sec}$ at the $1 / \mathrm{sec}, 2 / \mathrm{sec}$, and $4 / \mathrm{sec}$ rates, respectively. When expressed in terms of number of clicks, all three of these time values are virtually identical ( 9 to 10 clicks). The conclusion that number of clicks is the critical variable, which was supported in Figure 4 for the leftleading data, holds extremely well for the right-leading data at the longest delay.

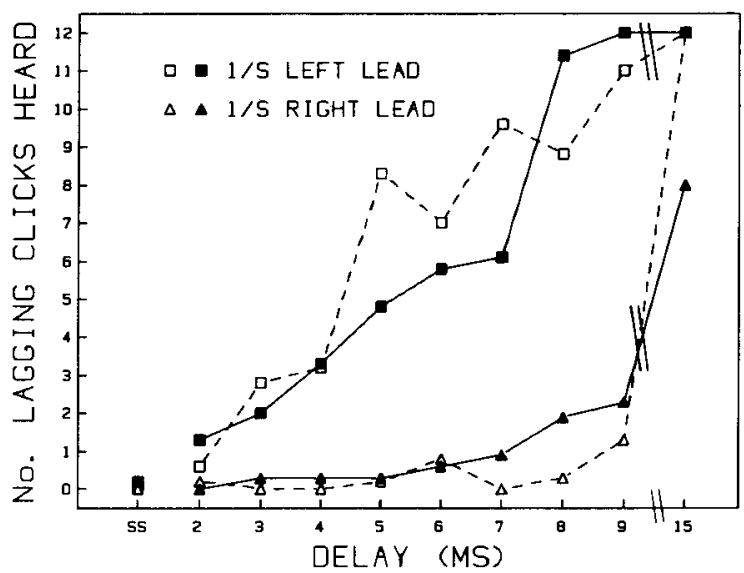

Figure 5. Effect of the leading click's location on the number of echo clicks heard following a switch in location for 2 subjects showing strong laterality. The dashed line represents Subject 1 and the solid line represents Subject 2 at the $1 /$ sec rate.

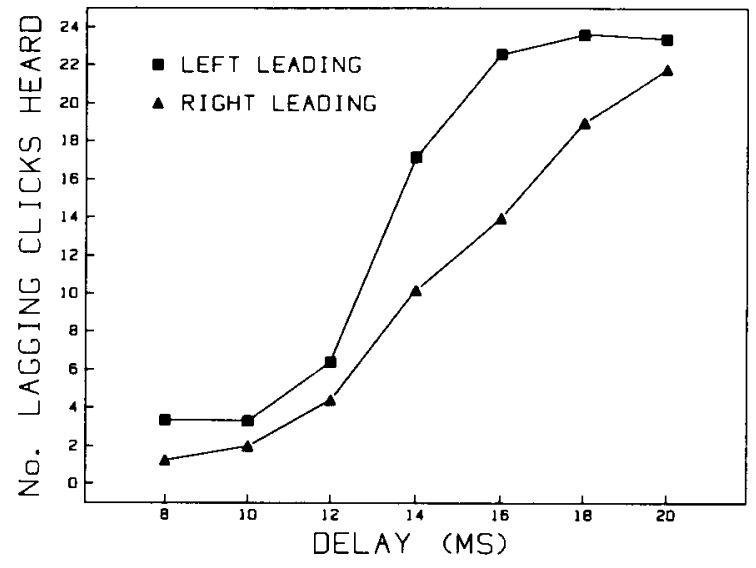

Figure 6. Effect of the leading click's location on the number of echo clicks heard following a switch in location. Data plotted are for all subjects at the $2 / \mathrm{sec}$ rate.

Because no data had been obtained between the 9- and the 15-msec delays, yet the perceptibility of the echo click changed so dramatically between those two conditions for both left- and right-leading clicks, it was decided that additional delays should be included to allow a more complete description of echo perceptibility over the longer delays. In addition, this would allow us to study the size of the laterality effect over a range of longer delays. All 5 subjects were tested at the $2 / \mathrm{sec}$ rate and 4 subjects were tested at the 4/sec rate with delays of $8,10,12,14,16$, 18 , and $20 \mathrm{msec}$ using identical click stimuli and procedures as those used in the main experiment. Each subject received a total of 84 trials at each rate, organized into 12 blocks of seven trials each ( 1 trial for each of the seven delays). Half the blocks were left-leading and half were right-leading; the order of blocks and delays within each block were random. The results for the $2 / \mathrm{sec}$ rate, with left- and right-leading clicks shown separately, are displayed in Figure 6. The number of lagging clicks heard increased smoothly with echo delay, but the function is steeper for the left-leading condition. The breakdown of echo suppression can occur as a result of a lead-lag switch in either direction, and the dependence on delay is qualitatively similar for both left- and right-leading clicks. However, longer delays were required to break down echo suppression, and suppression was reestablished faster when the right loudspeaker was leading after the switch. The curve for left-leading stimuli reaches asymptote around $16 \mathrm{msec}$, whereas the curve for right-leading stimuli reaches a similar level at $20 \mathrm{msec}$. Data for the 4/sec rate follow a similar pattern, showing a laterality effect most evident for delays between 14 and 20 msec.

Additional analysis of the subjects' responses suggests that one need not create an abrupt reversal in lead and lag locations in order to observe a buildup in suppression. Several subjects reported, both verbally and through their button presses, time-dependent changes in the perceptibility of the echo click in the 5-8-sec period of stimulation before the switch. These subjects indicated that on 
some trials, the echo click was clearly audible during the first few clicks immediately after onset of the stimulus train, then faded and was inaudible for the remainder of the time before the switch. This effect is similar to that reported by subjects in Thurlow and Parks's (1961) study.

Analysis of the preswitch data in our study was problematic because these click trains were of variable duration. This had the desired effect of preventing the subjects from anticipating the switch, but also made it difficult to combine preswitch results across trials. To facilitate at least some quantitative analysis, preswitch responses were assigned to one of three categories: (1) only the leadside button pressed, (2) both lead- and lag-side buttons pressed during any portion of the preswitch interval, and (3) both buttons pressed for the entire interval. The results were evaluated with regard to lagging-click delay, rate, and laterality. As expected, the number of trials on which responses fell into Categories 2 or 3 (some echo clicks were heard) was strongly dependent on delay. Click rate affected responses in the same direction as for the postswitch click train. For example, for left-leading clicks at the 9-msec delay, the subjects reported hearing echo clicks for the entire interval on 11 of 30 trials at the $1 / \mathrm{sec}$ rate, on 6 trials at the $2 / \mathrm{sec}$ rate, and on only 2 trials at the $4 / \mathrm{sec}$ rate. For right-leading clicks at the same delay, the subjects heard no echo clicks throughout the entire inter$\mathrm{val}$ at any rate. To the extent that the crude categorization of preswitch responses could be compared to postswitch responses, the same dependence on the variables of delay, rate, and laterality was observed.

\section{DISCUSSION}

Considering the long history of the precedence effect (see Gardner, 1968), it is surprising that more than 100 years of investigation passed before the slow-acting inhibitory process described here was noticed. The reason probably lies in methodology. Most research on the precedence effect has presented subjects with single brief stimuli on which to base their judgments of echo presence and/or location (see Zurek, 1987, for review). Under these experimental conditions, the subject has a brief opportunity to judge some aspect of the echo and to report this momentary perception at the end of the trial. A short exposure precludes the striking perception of the echo's being present and then fading away. The dynamic nature of echo suppression is revealed when listeners are presented with long click trains and are asked to evaluate the presence or absence of an echo click at its location. Continuous judgments allow listeners to update their perceptions moment by moment. Echo inhibition has been shown to be an inverse function of click delay and a direct function of number of clicks. These relationships might not hold over a wider range of delays and rates, but are true for at least a limited set of combinations. The general effect of click delay on echo inhibition is the same both for single clicks and for click trains; that is, longer delays lead to less, and eventually no, inhibition. The difference between single clicks and click trains lies in the final level of inhibition achieved at a particular delay. For single clicks, echoes are separately localized around 2 to $4 \mathrm{msec}$ (see Blauert, 1983, p. 231, Table 3.2, for a summary of experiments). For click trains of $5 / \mathrm{sec}$, the echo threshold is raised to 9 to $11 \mathrm{msec}$ (Thurlow \& Parks, 1961).

At very short delays, a single echo event produces inhibition. As delays are extended, more auditory events are required to produce inhibition. These events must occur within a limited time frame (the exact limits are unknown at this time); otherwise, each event will be acting alone, with no accumulation of inhibition. One can turn this argument around to predict that continuous longduration stimuli with numerous rapid transients would have higher echo thresholds than would short-duration stimuli with only a single transient. In explaining why a steady broadband noise was localized most easily, Hartmann (1983) hypothesized that "the binaural system regards noise to be a series of small impulses"' (p. 1388). Both steady noise and rapid click trains would produce greater echo suppression than would single clicks or steady sounds with few transients. The click train has an advantage over noise in that clicks can be delivered slowly enough to measure the buildup of inhibition as a function of number of clicks.

The contribution of rate (or number of clicks) may be more complex than our data currently show. Between the $1 / \mathrm{sec}$ and the $4 / \mathrm{sec}$ rates, we found that the number of clicks indicated the point at which echoes would be suppressed. Using a wider range of click rates, Thurlow and Parks (1961) reported a curvilinear effect. Comparing $1 / \mathrm{sec}$ to $5 / \mathrm{sec}$ rates, Thurlow and Parks found a lower threshold for the slower rate. However, a very fast rate of $50 / \mathrm{sec}$ also produced a lower threshold than did the $5 / \mathrm{sec}$ rate. Thurlow and Parks did not state the length of their click trains or the point at which their subjects were asked to make an echo-threshold judgment. Our data strongly indicate that both of these variables are critical, as echo perceptibility is affected by what has gone before. If echo suppression does not follow a monotonic curve indefinitely, but shows variation after a certain number of clicks, very fast rates could show a lowering of threshold after several seconds. This point would not be reached for slower rates unless click trains were presented for very long periods. The issue of how the number of clicks affects echo perceptibility across time is central to understanding the precedence effect, both at onset of click train and after the switch in location.

The buildup of echo suppression in a click train is influenced by delay and number of clicks, regardless of whether there is a switch in lead-lag location at some point. The unanswered question is to what extent the abrupt reversal in locations may decrease inhibition below the initial onset level, or simply "reset" the built-up inhibition to the same strength as at the beginning of the click train. In the former case, inhibition that was built up at the start of a click train may continue for a time after the switch, requiring several clicks from the new 
locations before the system recovers from the inhibition and recognizes fully that the former echo location is now the location of the primary sound source, and vice versa. Determining whether such a tendency toward enhanced echo perceptibility following reversal exists in the present data requires a quantitative comparison of pre- and postswitch responses which, as discussed above, is not possible with the procedure used in this study.

The laterality effect was unexpected because previous investigations of the precedence effect did not report greater inhibition of echoes from one side versus the other. Hartmann (1983) mentioned that 7 of 12 subjects had judgments of sound sources biased toward the left, in that errors in localization tended to be to the left, rather than to the right, of the correct position. Hartmann's task involved identifying the correct source out of an array of eight loudspeakers, and did not measure echo suppression similar to our task. Thus, it is difficult to compare his data with ours, except to note that a side bias was found. If one moves away from the precedence effect, evidence for laterality in auditory tasks abounds, and in the direction consistent with our data. Many auditory tasks, such as temporal-order threshold (Mills \& Rollman, 1980) and detection of rhythm patterns of tonal pulses (Gordon, 1978), show a right-ear advantage. The most extensive data have used verbal stimuli in dichotic listening experiments. Kimura (1967) proposed that separate but simultaneous inputs to the cerebral hemispheres would create competition, resulting in the dominant hemisphere's suppressing incoming information to the nondominant hemisphere. In dichotic listening experiments, subjects usually report input to the dominant hemisphere (typically the left in right-handed people) and fail to report input from the other side. Kimura stressed that a dichotic presentation was necessary to create the competition that resulted in cerebral dominance being shown. In the current study, stimuli were presented in free field, but competing localization cues were reaching the two ears: the leading click reached one ear first and the lagging click reached the opposite ear first. The precedence effect is the inhibition of the later-arriving localization information, so that the first-arriving cues are perceived as correct and are used to specify the location of the sound in space. If one hemisphere were better than the other at suppressing contralateral input, we would expect fewer echoes to be localized when input to the dominant hemisphere was leading. This prediction is upheld in our data; the left hemisphere apparently suppressed echoes better than did the right hemisphere. (We should note that all 5 subjects were right handed.) The precedence effect can be added to the list of temporal phenomena that show asymmetry in the processing of auditory input.

In conclusion, the most significant aspect of these data is that they may lead to a new conceptualization of the precedence effect as a dynamic process that is modified by previous and ongoing input. Current models of the precedence effect (see Lindemann, 1986; Zurek, 1987) postulate an inhibitory process or processes in which the portion of the auditory nervous system involved in localization weights the first-arriving information most heavily. Later-arriving directional information, whether it is based on monaural spectral cues, interaural time differences, or interaural intensity differences, is suppressed for a period of time after the arrival of the initial wavefront. Most data have been interpreted as indicating that the inhibitory process lasts for just a few milliseconds. These models predict the existing data on echo suppression when listeners are given a brief period to make a single judgment on each trial. The present results suggest that these models should be modified to include an additional slower dynamic process resulting from the localization of rapidly repeating transient stimuli that last several seconds.

\section{REFERENCES}

Blauert, J. (1983). Spatial hearing. Cambridge, MA: MIT Press. Clifton, R. K. (1987). Breakdown of echo suppression in the precedence effect. Journal of the Acoustical Society of America, 82, 1834-1835.

GARDNER, M. D. (1968). Historical background of the Haas and/or precedence effect. Journal of the Acoustical Society of America, 43, 1243-1248.

Gordon, H. W. (1978). Left hemisphere dominance for thythmic elements in dichotically presented melodies. Cortex, 14, 58-70.

HARTMANN, W. M. (1983). Localization of sound in rooms. Journal of the Acoustical Society of America, 74, 1380-1391.

KIMURA, D. (1967). Functional asymmetry of the brain in dichotic listening. Cortex, 3, 163-178.

LINDEMANN, W. (1986). Extension of a binaural cross-correlation model by contralateral inhibition: 2 . The law of the first wavefront. Journal of the Acoustical Society of America, 80, 1623-1630.

Mills, L., Rollman, G. B. (1980). Hemispheric asymmetry for auditory perception of temporal order. Neuropsychologia, 18, 41-47.

RAKERD, B., \& HARTMANN, W. M. (1985). Localization of sound in rooms: 2 . The effects of a single reflecting surface. Journal of the Acoustical Society of America, 78, 524-533.

RAKERD, B., \& HartmanN, W. M. (1986). Localization of sound in rooms: 3. Onset and duration effects. Journal of the Acoustical Society of America, 80, 1695-1706.

Thurlow, W. R., \& PARKs, T. E. (1961). Precedence-suppression effects for two click sources. Perceptual \& Motor Skills, 13, 7-12.

Wallach, H., Newman, E. B., \& Rosenzweig, M. R. (1949). The precedence effect in sound localization. American Journal of Psychology, 52, 315-336.

ZUREK, P. M. (1987). The precedence effect. In W. A. Yost \& G. Gourevitch (Eds.), Directional hearing (pp. 85-105). New York: Springer-Verlag.

(Manuscript received August 2, 1988; revision accepted for publication January $20,1989$. 